\title{
Métodos de diagnóstico e pontos críticos de bem-estar de bovinos leiteiros
}

\author{
Welfare assessment methods and critical points for dairy cattle
}

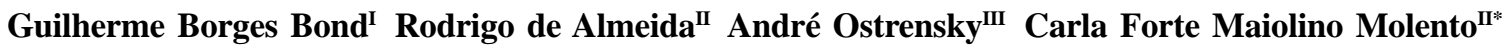

\section{- REVISÃO BIBLIOGRÁFICA -}

\section{RESUMO}

O bem-estar de animais de produção tem sido discutido nos âmbitos comercial, social e acadêmico, sendo que tal discussão pode ser enriquecida pela elaboração de protocolos de diagnóstico de bem-estar animal bem definidos, favorecendo a regulamentação de uma legislação específica. O objetivo desta revisão é discutir os métodos de diagnóstico de bem-estar animal e apontar os principais pontos críticos que afetam o bem-estar dos bovinos leiteiros. O diagnóstico de bem-estar compreende a observação do comportamento animal e de indicadores fisiológicos e sanitários, como análise hematológica e dosagem de hormônios, análise do escore de locomoção, a observação de lesões corporais e o estado geral de saúde dos animais. Tais ferramentas permitem verificar quais são os principais pontos críticos que afetam o bem-estar dos animais e construir estratégias de melhoria. As vacas em lactação podem sofrer restrições ambientais, nutricionais $e$ sanitárias. Os bezerros passam pelo distresse do desmame precoce e do isolamento social. Alguns pontos críticos de bemestar de gado leiteiro reconhecidos internacionalmente parecem prováveis no cenário brasileiro. Entretanto, é possível a existência de diferenças importantes em relação aos sistemas de produção praticados no Brasil e no exterior, uma vez que o acesso ao pasto é um fator comum na produção brasileira e pode estar associado a um maior grau de bem-estar animal. Dessa forma, é importante a condução de trabalhos de diagnóstico de bem-estar específico para os animais envolvidos com a produção de leite no Brasil.

Palavras-chave: estresse, leite, produção, vacas leiteiras.

\begin{abstract}
The welfare of farm animals is under broad discussion within the commercial, social and academic environments, and discussion may be enriched by specific guidelines and legislation based on well-defined assessment protocols. The objective of this review is to discuss the methods for assessing animal welfare and to point out the main critical points that affect the welfare of dairy cattle. The assessment of animal welfare is based on behavioral observations and physiological and health parameters such as analysis of blood parameters and hormone concentrations, locomotion scoring, presence of body lesions and general health state of the animals. These tools allow for the analysis of the main critical points that affect animal welfare and the proposal of improvement strategies. The lactating cows may suffer from environmental, nutritional and sanitary restrictions. Dairy calves go through weaning distress and social isolation. Some of the critical welfare points recognized internationally seems likely to occur in the Brazilian scenario. However, some differences possibly exist, once the access to pasture is a common factor in Brazilian production and may be associated with a higher welfare degree. Thus, it is important to carry on specific welfare assessment of the animals involved in milk production in Brazil.
\end{abstract}

Key words: stress, milk, production, dairy cows.

\section{INTRODUÇÃO}

Historicamente, o bem-estar dos animais de produção foi ofuscado pela busca de melhores índices

'Department of Production Animal Health, Faculty of Veterinary Medicine, University of Calgary, 3330 Hospital Dr. NW, T2N 4Z6, Calgary, Alberta Canadá.

"Departamento de Zootecnia, Centro de Ciências Agrárias,Universidade Federal do Paraná (UFPR), Rua dos Funcionários, 1540, 80035-050, Curitiba, PR, Brasil. E-mail: carlamolento@yahoo.com.*Autor para correspondência

II'Curso de Medicina Veterinária, Escola de Ciências Agrárias, Pontifícia Universidade Católica do Paraná, São José dos Pinhais, PR, Brasil. 
zootécnicos. Desde a década de 60, na União Europeia, por meio de iniciativas como o livro Animal Machines (HARRISON, 1964), a sociedade passou a conhecer os sistemas de produção animal e a exigir a criação de animais de maneira humanitária. Atualmente, produtos oriundos de sistemas de mais alto grau de bem-estar apresentam valores agregados, de ordem econômica e ética, atendendo à demanda de um nicho específico de mercado. Adicionalmente, a demanda social levou à elaboração de legislação específica a respeito do bemestar animal, a qual provavelmente originará o estabelecimento de barreiras comerciais entre países. Dessa forma, parece interessante o desenvolvimento de pesquisas na área de diagnóstico de bem-estar, para que se possa subsidiar a elaboração de leis e o controle do bem-estar animal nos sistemas produtivos.

A disponibilidade de área e o clima no território nacional tornam viável a criação de animais a pasto. Tal sistema, por apresentar menor restrição comportamental, está potencialmente relacionado a vantagens para o bem-estar animal, desde que sejam utilizadas práticas de manejo e controle sanitário adequadas. O equilíbrio final entre desafios e vantagens para na pecuária leiteira nacional, que compõe, em última instância, a qualidade de vida dos animais, ainda é desconhecido pela escassez de publicações nesta área no Brasil. O objetivo desta revisão é apresentar os métodos de diagnóstico de bem-estar animal utilizados mundialmente e apontar os principais pontos críticos que afetam os bovinos leiteiros no Brasil, bem como a aplicabilidade de tais métodos diagnósticos na pecuária leiteira brasileira.

Métodos de diagnóstico de bem-estar de bovinos leiteiros

O bem-estar animal é definido como o estado do animal frente às suas tentativas de se adaptar ao ambiente em que se encontra (BROOM, 1986). Portanto, quanto maior o desafio imposto pelo ambiente, mais dificuldade o animal terá em se adaptar e, consequentemente, menor será seu grau de bemestar. A avaliação científica do bem-estar deve considerar o estado do animal de forma objetiva e separada de questões éticas (BROOM \& MOLENTO, 2004). Para conhecer de maneira científica o grau de bem-estar animal é necessário o desenvolvimento de técnicas de diagnóstico. Nos procedimentos de diagnóstico centrados no animal, os indicadores mais utilizados são as respostas fisiológicas e comportamentais e a sua condição sanitária (LEEB et al., 2004). Outra abordagem centrada no animal é representada pelo trabalho de DUNCAN (2005), que considera a avaliação das emoções dos animais como a parte principal do diagnóstico de bem-estar animal. Apesar de tal avaliação ser subjetiva e de difícil aplicação prática, ela é central do ponto de vista do bem-estar dos animais.

A fisiologia também constitui importante instrumento nesse diagnóstico. Dentre as medidas fisiológicas diretas, estão aquelas decorrentes da ativação do Sistema Nervoso Autônomo (SNA), como alterações das frequências cardíaca e respiratória. Tais medidas estão associadas a respostas de curto prazo e são mensuráveis de maneira objetiva (BROOM \& JOHNSON, 1993). Por exemplo, COLLIER et al. (1982) alojaram vacas no final da gestação em piquetes com e sem sombreamento. Os animais alojados sem sombra apresentaram maiores frequências cardíaca e respiratória, revelando ativação do SNA em condições climáticas adversas e menor grau de bem-estar animal. Trabalhos mais recentes, como o de VAN REENEN et al. (2002), detectaram alterações na frequência cardíaca de algumas vacas primíparas no início da lactação, quando eram apresentadas ao sistema de ordenha mecânica.

Assim como na resposta do SNA, é possível detectar alterações na concentração de hormônios adrenocorticais como consequência de estímulos adversos. Nesse contexto, o indicador mais utilizado nos ruminantes é o nível de cortisol. A dosagem de cortisol plasmático vem sendo utilizada para se analisar os efeitos de curto prazo de práticas de manejo sobre o bem-estar animal (BROOM \& FRASER, 2007). RUSHEN et al. (2001) estudaram os efeitos do estresse agudo ocasionado por isolamento e ordenha em ambientes desconhecidos sobre o perfil endócrino de vacas leiteiras; tais condições induziram concentrações plasmáticas de cortisol significativamente superiores em relação ao grupo controle.

A dosagem de cortisol nas fezes surgiu como um método não invasivo para a sua mensuração (PALME et al., 1999). Em ruminantes, o cortisol liberado na corrente sanguínea atinge o trato intestinal em aproximadamente 12 horas (PALME et al., 1996). Portanto, para fins de avaliação do cortisol plasmático, tal cronologia deve ser considerada. Pode-se concluir que os parâmetros fisiológicos são importantes para o diagnóstico de bem-estar animal. Porém, existem limitações para a sua aplicação na rotina de diagnóstico de campo, especialmente quanto aos cuidados na colheita de amostras e aos seus custos. Dessa forma, há necessidade de seleção de indicadores fisiológicos práticos, que permitam utilização em campo e que, associados a outras mensurações práticas, reflitam de maneira eficiente o grau de bem-estar dos animais. 
O comportamento também constitui importante instrumento no diagnóstico de bem-estar animal. O conhecimento do comportamento natural do animal é importante para se diagnosticar e aprimorar seu grau de bem-estar (FRASER, 1993). Por exemplo, alterações de postura, locomoção e temperamento, aliados a observações do estado sanitário, podem indicar que um animal sente dor, bem como quantificála(MELLOR \& STAFFORD, 2004).

As diferentes espécies animais apresentam comportamentos que podem ser descritos como sendo de alta motivação, ou seja, atividades tidas como prioritárias pelo sistema de controle cerebral (BROOM \& JOHNSON, 1993). Em ambientes artificiais que restrinjam comportamentos de alta motivação, o grau de bem-estar do animal encontra-se reduzido (DUNCAN, 1998). No caso dos bovinos, um exemplo seria a restrição do comportamento de sugar dos bezerros. As bezerras leiteiras, quando aleitadas por meio de baldes, são privadas do comportamento de sugar as tetas da mãe. As consequências de tal privação são o aumento da frequência de comportamento oral estereotípico (BERGERON et al., 2006), caracterizado por sugar outras bezerras e partes das instalações, e rolar de língua, aliados à possível redução no ganho de peso (DE PASSILLÉ, 2001). Ainda, a presença de comportamento estereotípico constitui um bom indicador de redução no grau de bem-estar animal (BROOM \& JOHNSON, 1993). Outros exemplos de comportamentos de alta motivação que podem ser restritos em sistemas produtivos são o pastejo, a relação mãe-filho e a formação de grupos sociais naturais. Ainda, a interação ser humano-animal tem grande influência sobre o comportamento animal, podendo limitar seu bem-estar e sua produtividade (HEMSWORTH \& COLEMAN, 1998). Medidas de comportamento, como a distância de fuga, podem ser utilizadas como indicadores da qualidade do manejo na propriedade; porém, necessitam de maior padronização entre os trabalhos científicos para que se tornem mais confiáveis(DE PASSILLÉ\& RUSHEN, 2005).

Alguns protocolos de diagnóstico consideram fatores como instalações, piquetes, sistemas de ventilação e de sombreamento como pontos importantes para se determinar o potencial de bemestar animal de um sistema produtivo (BARTUSSEK et al., 2000; BCSPCA, 2001; NDFAS, 2004; RSPCA, 2008). O Índice de Necessidades dos Animais (BARTUSSEK et al., 2000) baseia-se na análise da adequação das instalações em relação às necessidades do animal. São observados aspectos como facilidade de locomoção e de interações sociais, tipo e condição do piso, ventilação, iluminação e manutenção das instalações.
Ainda, tal índice considera aspectos observados diretamente nos animais, como sua saúde e integridade. Alguns protocolos também consideram as condições de transporte e abate emergencial de animais (RSPCA, 2008).

Todas as ferramentas e tipos de abordagem citados podem ser combinados em protocolos que permitam diagnosticar o bem-estar dos animais de maneira prática, eficiente e fidedigna à realidade das diferentes propriedades.

Pontos críticos de bem-estar animal na produção leiteira GREGORY (1998) enumera pontos críticos de bem-estar na produção leiteira. Alguns são inerentes aos sistemas, como restrições comportamentais decorrentes do confinamento. Outros podem ser considerados pontos críticos evitáveis, como a subnutrição. MOLENTO \& BOND (2008) propõem a adição de outros fatores à lista de GREGORY (1998), considerando o contexto brasileiro. As vacas leiteiras brasileiras têm, em geral, acesso a pasto; tal fato favorece potencialmente um maior grau de bem-estar, desde que sombreamento seja disponível e que outros problemas evitáveis sejam resolvidos.

Um ponto crítico discutido em livros-texto é a pressão para mais alta produção, sendo a crescente seleção para produção de leite um dos exemplos citados (BENSON \& ROLLIN, 2004; WEBSTER, 2005). OLTENACU\& ALGERS (2005) relatam que a seleção genética para alta produção leva a decréscimo de fertilidade e longevidade das vacas, além de elevar os índices de doenças inerentes à produção. A difusão de material genético proveniente da Europa e principalmente da América do Norte foi um dos fatores responsáveis pelo aumento da produtividade das fazendas leiteiras no Brasil. Entretanto, tal processo gerou animais pouco adaptados a climas quentes. As dificuldades relacionadas à adaptação ao clima e mais alta demanda nutricional dos animais de alta produção, associadas ao menor ritmo de alterações de manejo, podem reduzir severamente o grau de bem-estar dos animais. A pressão por produção é ainda mais intensa quando se introduz a utilização da somatotropina bovina, em animais de produção intrinsecamente alta (BROOM \& FRASER, 2007). O Comitê Científico de Sanidade e Bem-estar Animal da União Europeia relata os efeitos nocivos da utilização indiscriminada de tal hormônio, descrevendo um aumento significativo na incidência de doenças no rebanho (EUSCAHAW, 1999).

A consideração da prevalência de doenças no rebanho leiteiro também compõe a identificação dos pontos críticos de bem-estar animal. O registro da prevalência de doenças e dos tratamentos realizados permite ações preventivas de controle das principais 
enfermidades locais (WHAY et al., 2003). Aidentificação de vacas em risco de apresentar doenças pode diminuir a incidência de enfermidades. Por exemplo, observouse que vacas com maior risco de desenvolver metrite pós-parto apresentaram redução mais severa da duração de comportamento ingestivo nos dias imediatamente anteriores ao parto (URTON et al., 2005). Assim, alterações na ingestão de matéria seca podem ser utilizadas para identificar vacas predispostas a metrite e outras doenças comuns ao período de transição (HUZZEY et al., 2005).

Algumas doenças são especialmente relevantes para o diagnóstico de bem-estar de gado leiteiro. A mastite continua apresentando alta incidência, mesmo após o desenvolvimento de técnicas preventivas, dentre elas a melhoria na higiene de ordenha (BRADLEY, 2002). Como a mastite é um processo inflamatório, o animal está sujeito à dor desencadeada pela liberação de mediadores inflamatórios. ESHRAGHI et al. (1999) identificaram altas concentrações de bradicinina no leite de vacas afetadas por mastite. Levando em conta a importância do binômio intensidade e duração para o diagnóstico de bem-estar animal (BROOM \& JOHNSON, 1993), pode-se concluir que um animal apresentando mastite severa por longo período tem seu grau de bem-estar reduzido de maneira importante.

De maneira semelhante à mastite, as afecções do casco dos bovinos apresentam-se em altas incidências. A claudicação é um sinal clínico comum a várias doenças que afetam o casco dos bovinos (GREENOUGH, 1997). O animal afetado distribui o peso corporal de maneira desigual entre os quatro membros, levando a alterações na locomoção. Animais com claudicação severa evitam apoiar o membro afetado no chão, causando um maior desequilíbrio locomotor e sobrecarregando os outros membros menos afetados (NEVEUX et al., 2006). Além disso, poucos produtores adotam técnicas de diagnóstico e controle de claudicação (STOKES et al., 2008), sendo que WHAY et al. (2002) relataram que a incidência de claudicação é subestimada pelos produtores. Também existe a tendência de se tratar somente os casos de claudicação evidente, deixando de lado animais que também se beneficiariam do tratamento, mas que não apresentam sinais tão severos.

A dor é uma característica comum da maioria das doenças do casco. Testes de sensibilidade à dor demonstram que um dos efeitos de lesões na camada córnea do casco é a diminuição no limiar de estímulo doloroso do animal (WHAY et al., 1997). Por menor que seja a lesão, a inflamação leva à sensibilização do membro afetado. GREENOUGH (1997) avalia os efeitos da claudicação sobre o bem-estar animal de maneira mais ampla, considerando o conceito das cinco liberdades (WEBSTER, 2005) e afirmando que todas elas são afetadas negativamente quando o animal é acometido por doenças do casco.

Outras doenças da produção também afetam o bem-estar animal. MULLIGAN \& DOHERTY (2008) definem tais doenças como sendo consequências de um nível de produção inconsistente com aquele da ingestão de nutrientes. Tais doenças são muito prevalentes nos sistemas de produção leiteira, podendo afetar um número considerável de animais por longos períodos durante o ciclo produtivo e aumentar a taxa de descarte involuntário de animais. WEBSTER (2005) relata que, na maioria das propriedades leiteiras americanas e britânicas, a longevidade média de uma vaca leiteira é inferior a três lactações, sendo que a maioria dos descartes não ocorre voluntariamente, mas por fatores como mastite, claudicação e infertilidade. SILVA\& ALMEIDA (2009) identificaram que as principais causas de descarte involuntário na região de Arapoti, Paraná, consistem em problemas reprodutivos, mastite e alta contagem de células somáticas, seguidos de doenças podais; os autores ainda observaram que propriedades com menos vacas descartaram menos animais. Tal resultado foi atribuído ao manejo mais individualizado de animais nessas propriedades. A nutrição da vaca leiteira atual também representa um potencial ponto crítico de bem-estar. As exigências nutricionais de vacas leiteiras foram consideravelmente aumentadas nas últimas décadas, pela exacerbação de sua capacidade produtiva por meio da seleção artificial. Animais que se encontrem impossibilitados de ingerir uma quantidade adequada de nutrientes, seja por questões ambientais ou desproporcionalidade entre trato digestivo e necessidades nutricionais, podem sofrer fome crônica e doenças metabólicas, principalmente no primeiro terço da lactação.

Alguns fatores adicionais devem ser levados em consideração. Foi constatado que, em instalações com menos de $60 \mathrm{~cm}$ de cocho disponível por animal, menos de 70\% das vacas conseguem acesso ao cocho simultaneamente (DEVRIES et al., 2003). Tais resultados sugerem que alguns animais tendem a ter sua ingestão de alimentos limitada. DEVRIES \& VON KEYSERLINGK (2006) concluíram que um espaço maior no cocho faz com que a intensidade de interações agressivas entre os animais diminua e o tempo que os animais passam se alimentando aumente. Outros trabalhos demonstraram que as vacas aumentam a frequência de alimentação em ambientes que limitam os comportamentos competitivos (HUZZEY et al., 2005; 
DEVRIES et al., 2005). No Brasil, existe o fator da escassez estacional de forragem. Em algumas regiões, a produção constante de alimento de qualidade é dificultada por períodos de seca intensa, o que faz com que a disponibilidade de forrageiras tropicais varie muito durante o ano.

Outro fator que pode diminuir o grau de bem-estar animal é o confinamento, que pode ser agravado por projetos inadequados. WEBSTER (1986) ressalta que as vacas necessitam de instalações limpas e confortáveis, de fácil acesso, nas quais possam se deitar e levantar com facilidade, além de manter o contato social com outras vacas. Trabalhos mais recentes procuraram identificar qual o material da superfície da baia mais confortável para os animais (TUCKER \& WEARY, 2004), entender as preferências quanto ao tipo de piso (STEFANOWSKA et al., 2002) e monitorar diversos índices de conforto em vacas alojadas em estabulação livre (COOK et al., 2005). Um projeto inadequado das instalações pode ter consequências diretas em termos de lesões nos membros dos animais (CEBALLOS et al., 2004), também denominadas tecnopatias, seja pela má distribuição de peso sobre eles ou por lesões de abrasão nas estruturas da baia. WEARY \& TASZKUN (2000) demonstraram que alguns tipos de cama podem predispor a lesões de jarrete. A quantificação de tecnopatias é importante para a avaliação de restrições da liberdade de desconforto e de ferimentos.

O projeto das baias e da drenagem de dejetos dos corredores da estabulação livre exerce influência direta sobre a saúde do úbere (TUCKER et al., 2003). As vacas evitam usar baias desconfortáveis, preferindo deitar-se nos corredores sujos com fezes, aumentando o risco de mastites. Adicionalmente, um material que dificulte a limpeza das camas aumenta o risco de mastites ambientais (ZDANOWICZ et al., 2004). A manutenção da baia e a reposição do material da cama também são importantes. Uma das principais consequências de falhas na manutenção das baias é a redução do tempo de descanso dos animais (DRISSLER et al., 2005). Tal diminuição de tempo de descanso constitui uma redução direta do grau de bem-estar dos animais. Além disso, a relutância em se deitar pode apresentar implicações indiretas, como aumentar a incidência ou a severidade de claudicação.

O acesso ao pasto é um fator importante para o comportamento e o bem-estar dos bovinos leiteiros. REGULA et al. (2004) testaram o efeito de diferentes sistemas de produção sobre o bem-estar e a saúde das vacas. Os autores concluíram que o acesso às áreas externas tem efeitos positivos sobre o bemestar de vacas confinadas. Estudos de preferência, como de LEGRAND et al. (2009), indicam que a preferência dos animais pelo pasto ou pelo galpão de confinamento pode estar condicionada a vários fatores, como ao período do dia e fatores ambientais. Os autores encorajam futuros trabalhos que considerem fatores adicionais como a qualidade das instalações internas e externas, bem como a experiência prévia dos animais em diferentes ambientes. Apesar de a preferência variar entre galpão e ar livre ao longo do dia, a liberdade de escolha do animal constitui um aspecto positivo para seu bem-estar (FRASER \& MATTHEWS, 1997). No Brasil, ao contrário dos principais países europeus e norte-americanos, o sistema de semiconfinamento é difundido. Nesse sistema, os animais permanecem confinados a maior parte do tempo, porém são soltos a pasto em determinadas horas do dia. Tal prática pode representar uma importante vantagem em termos de bem-estar animal, como a manutenção da saúde do casco (OLMOS et al., 2009) e a possibilidade do animal de exercer comportamentos como o pastejo e interações sociais com outros membros do rebanho (HEMSWORTH et al., 1995). Além disso, um recente estudo demonstra que o acesso ao pasto durante o período noturno não representa prejuízos na produção ou ingestão de matéria seca (CHAPINAL et al., 2010)

Os problemas de bem-estar enfrentados pelos animais jovens são de importância especial na bovinocultura de leite. A separação precoce da vaca e do bezerro é um processo estressante para ambos, sendo um ponto crítico de bem-estar (GREGORY, 1998). Outro fator importante no manejo de bezerras leiteiras são os procedimentos de mutilação, como a descorna. Existem diferentes métodos de descorna, como a curetagem, o uso de serra, cisalhamento, ferro quente e pasta cáustica, que são dolorosos para os animais (SYLVESTER et al., 1998). Estudos realizados por FAULKNER \& WEARY (2000) testaram o efeito da administração de um antiinflamatório não esteroidal (cetoprofeno) antes da descorna sobre a expressão de comportamento de dor e desempenho das bezerras. Os resultados demonstraram que os animais tratados apresentaram uma queda significativa na frequência de comportamentos de dor e um maior ganho de peso nos dias subsequentes à descorna, demonstrando que o uso de tal medicamento mostrou-se eficiente no combate à dor após a descorna. Um ponto crítico pouco discutido na literatura é o bem-estar de bezerros machos. Como não podem ser inseridos no ciclo produtivo para reposição, os machos são tratados como um subproduto do sistema de produção. As alternativas mais utilizadas atualmente são a criação de vitelo ou o abate na primeira semana de vida, assim como a cria para consumo na própria fazenda. A 
produção de vitelo nos métodos tradicionais oferece restrições severas ao bem-estar dos animais. Métodos alternativos foram desenvolvidos, com melhorias ao bem-estar dos animais, como alojamento em grupos e fornecimento de dieta sólida (XICCATO et al., 2002). Porém, existem poucos estudos quanto à viabilidade de tais sistemas no contexto brasileiro (COSTA et al., 2001; ROMA JUNIOR. et al., 2008), sendo que as questões relativas ao bem-estar não são abordadas ou são brevemente mencionadas.Os animais vendidos para o abate na primeira semana de vida podem sofrer restrições de bem-estar, principalmente devido ao baixo preço pago por eles. Por serem animais de baixo valor e que não geram lucro direto à propriedade, na prática, costumam ser relegados a um segundo plano. Os bezerros podem sofrer restrições alimentares, algumas vezes, severas e podem ser alojados em ambientes estéreis e pouco confortáveis até o momento do transporte. Uma vez que o bezerro é gerado no contexto da atividade leiteira, o seu bem-estar é de responsabilidade de todos aqueles envolvidos na cadeia produtiva, incluindo produtores, responsáveis técnicos, processadores, comerciantes e consumidores.

\section{CONCLUSÃO}

Os métodos de diagnóstico permitem evidenciar os principais pontos críticos do bem-estar de gado leiteiro nos países europeus e norteamericanos e fazer algumas inferências para a realidade das propriedades leiteiras no Brasil. Análises das instalações e dos recursos fornecidos aos animais podem ser úteis na avaliação dos sistemas produtivos brasileiros, visto que, nas regiões de mais alta produção por animal, adotam-se projetos de instalações muito semelhantes aos europeus. O sistema de semiconfinamento aparece como um ponto positivo na produção brasileira. Pontos críticos podem ser apontados em diversas áreas da produção, como na criação de bezerras de reposição e no tratamento dispensado aos bezerros machos. Ainda, a adequação genética dos animais torna difícil sua adaptação a climas muito quentes ou que apresentem flutuação na disponibilidade de forragem. Uma parte de tais pontos críticos é evitável, principalmente quando envolve a falta ou má distribuição de recursos aos animais. Outros, como a seleção genética e a separação precoce dos bezerros, são menos passíveis de mudança imediata, visto que são intrínsecos aos sistemas de produção tradicionalmente utilizados. Futuros trabalhos são necessários para se elaborar protocolos de diagnóstico de bem-estar com indicadores aplicáveis a diversos sistemas de produção distintos e adequados ao contexto brasileiro. O desenvolvimento de tais protocolos depende de estudos básicos como estudos epidemiológicos de claudicação e o levantamento das condições das instalações de confinamento nas diferentes regiões brasileiras. Assim, permite-se que sejam desenvolvidas técnicas de diagnóstico específicas para a realidade produtiva brasileira, que subsidiarão protocolos de certificação e o desenvolvimento de legislação pertinente às características da produção leiteira nacional.

\section{REFERÊNCIAS}

BARTUSSEK, H. et al. Animal needs index for cattle. Gumpenstein: Federal Research Institute for Agriculture in Alpine Regions, 2000. 20p.

BCSPCA. Certified Standards for the raising and handling of dairy cattle. Vancouver: British Columbia Society for the Prevention of Cruelty to Animals, 2001. 19p.

BENSON, G.J.; ROLLIN, B. The well-being of farm animals: challenges and solutions. Oxford: Blackwell Publishing, 2004. 356p.

BERGERON, R. et al. Stereotypic oral behaviour in captive ungulates: foraging, diet and gastrointestinal function. In: MANSON, G.; RUSHEN, J. Stereotypic animal behaviour: fundamentals and application to welfare. Wallingford: CABI, 2006. p19-57.

BRADLEY, A.J. Bovine mastitis: an evolving disease. Veterinary Journal, Londres, v.164, p.116-128, 2002.

BROOM, D.M.; FRASER, A.F. Domestic animal behavior and welfare. Cambridge: CABI, 2007. 438p.

BROOM, D.M.; JOHNSON, K.G. Stress and animal welfare. Londres: Lower Academic, 1993. 228p.

BROOM, D.M.; MOLENTO, C.F.M. Bem-estar animal: conceito e questões relacionadas- revisão. Archives of Veterinary Science, Curitiba, v.9, p.1-11, 2004.

CEBALLOS, A. et al. Improving stall design: Use of 3-D Kinematics to measure space use by dairy cows when lying down. Journal of Dairy Science, Champaign, v.87, p.20422050, 2004

CHAPINAL, N. et al. Overnight access to pasture does not reduce milk production or feed intake in dairy cattle. Livestock Science, Elmsford, v.129, p.104-110, 2010.

COLLIER, R.J. et al. Effects of heat stress during pregnancy on maternal hormone concentrations, calf birth weight and postpartum milk yield of Holstein cows. Journal of Animal Science, Champaign, v.54, p.309-319, 1982.

COOK, N.B. et al. Monitoring indices of cow comfort in freestall-housed dairy herds. Journal of Dairy Science, Champaign, v.88, p.3876-3885, 2005. 
COSTA, F.P. et al. Carne de vitelão: estudo exploratório de um mercado em potencial. Campo Grande: EMBRAPA Gado de Corte, 2001. 21p.

DE PASSILLÉ, A.M.; RUSHEN, J. Can we measure humananimal interactions in on-farm animal welfare assessment? Some unresolved issues. Applied Animal Behavior Science, Amsterdam, v.92, p.193-209, 2005.

DE PASSILLÉ, A.M. Sucking motivation and related problems in calves. Applied Animal Behavior Science, Amsterdam, v.72, p.175-187, 2001.

DEVRIES, T.J. et al. Diurnal feeding pattern of lactating dairy cows. Journal of Dairy Science, Champaign, v.86, p.40794082, 2003.

DEVRIES, T.J. et al. Frequency of feed delivery affects the behavior of lactating dairy cows. Journal of Dairy Science, Champaign, v.88, p.3553-3562, 2005.

DEVRIES, T.J.; VON KEYSERLINGK, M.A.G. Feed stalls affect the social and feeding behavior of lactating dairy cows. Journal of Dairy Science, Champaign, v.89, p.3522-3531, 2006.

DRISSLER, M. et al. Free stall maintenance: effects on lying behavior of dairy cattle. Journal of Dairy Science, Champaign, v.88, p.2381-2387, 2005.

DUNCAN, I.J.H. Behavior and behavioral needs. Poultry Science, Champaign, v.77, p.1766-1772, 1998.

DUNCAN, I.J.H. Science-based assessment of animal welfare: farm animals. Revue Scientifique et Technique Office International des Epizooties, Paris, v.2, n.24, p.483-492, 2005.

ESHRAGHI, H.R. et al. The release of bradykinin in bovine mastitis. Life Sciences, Elmsford, v.64, n.8, p.1675-1687, 1999.

EUSCAHAW. Report on animal welfare aspects of the use of bovine somatotrophin. Bruxelas, 1999. 91p.

FAULKNER, P.M.; WEARY, D.M. Reducing pain after dehorning in dairy calves. Journal of Dairy Science, Champaign, v.83, p.2037-2041, 2000.

FEIJÓ, G.L.D. et al. Carne de vitelão: estudo exploratório de um mercado potencial. Campo Grande: Embrapa Gado de Corte, 2001. 21p. (Embrapa Gado de Corte - Documentos, 105).

FRASER, D. Assessing animal well-being: common sense, uncommon science. In: ALBRIGHT, J.L. (Ed.). Food animal well-being. West Lafayette, USDA: Purdue University, 1993. p.37-54.

FRASER, D.; MATTHEWS, L.R. Preference and motivation testing. In: APPLEBY, M.C.; HUGHES, B.O. Animal welfare. Wallingford: CABI, 1997. p.159-173.

GREENOUGH, P.R. Lameness in cattle. Philadelphia: Saunders, 1997. 277p.

GREGORY, N.G. Animal welfare and meat science. Londres: CABI Publishing, 1998. 304p.
HARRISON, R. Animal machines: the new factory farming industry. Londres: Vincent Stuart, 1964. 215p.

HEMSWORTH et al. The welfare of extensively managed dairy cattle: a review. Applied Animal Behaviour Science, Amsterdam, v.42, p.161-182, .1995

HEMSWORTH, P.H.; COLEMAN, G.J. Human-livestock interactions: the stockperson and the productivity and welfare of intensively-farmed animals. Oxford: CAB International, 1998. 208p.

HUZZEY, J.M. et al. Changes in feeding, drinking and standing behavior of dairy cows during the transition period. Journal of Dairy Science, Champaign, v.88, p.2454-2461, 2005.

IBGE. Pesquisa trimestral do abate de animais. Disponível em: <http://www.sidra.ibge.gov.br/>. Acesso em: 01 abr. 2011.

LEEB, C. et al. Bristol welfare assurance programme: cattle assessment. Bristol: University of Bristol, 2004. 17p.

LEGRAND, A.L. et al. Preference and usage of pasture versus free-stall housing by lactating dairy cattle. Journal Dairy Science, Champaign, v.92, p.3651-3658, 2009.

MAIN, D.C.J. et al. Bristol welfare assurance programme: animal based assessment tool for farm animal welfare certification. Bristol: University of Bristol, 2004. Disponível em: <http://www.vetschool.bris.ac.uk/animalwelfare/images/ BWAPweboverview.pdf $>$. Acesso em: 2 jan. 2008.

MELLOR, D.J.; STAFFORD, K.J. Physiological and behavioural assessment of pain in ruminants: principles and caveats. ATLA Fourth World Congress, v.32, supl.1, p.267271, 2004.

MOLENTO, C.F.M.; BOND, G.B. Aspectos éticos e técnicos da produção de bovinos. In: CONGRESSO BRASILEIRO DE BIOÉTICA E BEM-ESTAR ANIMAL, 2008, Recife, PE. Anais... Recife: Conselho Federal de MedicinaVeterinária, 2008. p.43-48.

MULligAN, F.J.; DOHERTY, M.L. Production diseases of the transition cow. Veterinary Journal, Londres, v.176, p.3-9, 2008.

NATIONAL DAIRY FARM ASSURED. Standards \& guidelines for assessment. Dumfries, 2004. 34p.

NEVEUX, S. et al. Hoof discomfort changes how dairy cattle distribute their body weight. Journal of Dairy Science, Champaign, v.89, p.2503-2509, 2006.

OLMOS, G. et al. Hoof disorders, locomotion ability and lying times of cubicle-housed compared to pasture-based dairy cows. Livestock Sicence, Elmsford, v.125, p.199-207, 2009.

OLTENACU, P.A.; ALGERS, B. Selection for increased production and the welfare of dairy cows: are new breeding goals needed? AMBIO, v.34, n.4, p.311-315, 2005.

PALME, R. et al. Excretion of infused ${ }^{14} \mathrm{C}$-steroid hormones via faeces and urine in domestic livestock. Animal Reproduction Science, Amsterdam, v.43, p.43-63, 1996. 
PALME, R. et al. Measurement of faecal cortisol metabolites in ruminants: a non-invasive parameter of adrenal function. Wiener TierärzlicheMonatsschrift, v.86, p.237-241, 1999.

REGULA, G. et al. Health and welfare of dairy cows in different husbandry systems in Switzerland. Preventive Veterinary Medicine, v.66, n.4, p.247-264, 2004.

ROMA JUNIOR, L.C. et al. Produção de vitelos a partir de bezerros leiteiros mestiços e da raça Holandesa. Revista Brasileira de Zootecnia. Viçosa, v.37, n.6, p.1088-1093, 2008.

RSPCA - Welfare Standards for dairy cattle. West Sussex: RSPCA Freedom Food, 2008. 53p.

RUSHEN, J. et al. Human contact and the effects of acute stress on cows at milking. Applied Animal Behaviour Science, Amsterdam, v.73, p.1-14, 2001.

SILVA, D.F.F.; ALMEIDA, R. Principais causas de descarte de vacas leiteiras na região de Arapoti, Paraná. In: REUNIÃO ANUAL DA SOCIEDADE BRASILEIRA DE ZOOTECNIA, 46. 2009, Maringá. Anais... Maringá: SBZ, 2009. Disponível em: < h t t p : / / w w w. hol andesparana.com.br/artigos/ artapcbrhdescarte.pdf>. Online. Acesso em: 18 jun. 2009.

STEFANOWSKA, J. et al. Do cows prefer a barn compartment with a grooved or slatted floor? Journal of Dairy Science, Champaign, v.85, p.79-88, 2002.

STOKES, J. et al. Lameness in dairy cattle: Relationships between environments, animal based welfare measures and locomotion. In: INTERNATIONAL SYMPOSIUM ON LAMENESS IN RUMINANTS, 15., 2008, Kuopio, Finlândia. Proceedings...Kuopio: Savonia University of Applied Sciences, 2008. p.49-52.

SYLVESTER, S.P. et al. Acute cortisol responses of calves to four methods of dehorning by amputation. Australian Veterinary Journal, Saint Leonards, v.76, p.123-126, 1998.

TUCKER, C.B. et al. Effects of three types of free-stall surfaces on preferences and stall usage by dairy cows. Journal of Dairy Science, Champaign, v.86, p.521-529, 2003.

TUCKER, C.B.; WEARY, D.M. Bedding on geotextile mattresses: how much is needed to improve cow comfort.
Journal of Dairy Science, Champaign, v.87, p.2889-2895, 2004.

URTON, G. et al. Feeding behavior identifies dairy cows at risk for metritis. Journal of Dairy Science, Champaign, v.88, p.2843-2849, 2005.

VAN REENEN, C.G. et al. Individual differences in behavioural and physiological responsiveness of primiparous dairy cows to machine milking. Journal of Dairy Science, Champaign, v.85, p 2551-2561, 2002.

WEARY, D.M.; TASZKUN, I. Hock lesions and free-stall design. Journal of Dairy Science, Champaign, v.83, p.697-702, 2000.

WEBSter, A.J.F. Animal welfare: limping towards eden. 2.ed. Oxford: Blackwell Publishing, 2005. 283p.

WEBSTER, A.J.F. Health and welfare of animals in modern husbandry systems - dairy cattle. In Practice, Londres, v.8, p.85-89, 1986.

WHAY, H.R. et al. Assessment of the welfare of dairy cattle using animal-based measurements: direct observations and investigation of farm records. Veterinary Record, Londres, v.153, n.7, p.197-202, 2003.

WHAY, H.R. et al. Associations between locomotion, claw lesions and nociceptive thresholds in dairy heifers during the peri-partum period. Veterinary Journal, Londres, v.154, p.155-161, 1997.

WHAY, H.R. et al. Farmer perception of lameness prevalence. In: INTERNATIONAL SYMPOSIUM LAMENESS IN RUMINANTS, 12., 2002, Orlando, Florida. Proceedings... Orlando: Jan K. Shearer 2002. 507p. p.355-358.

XICCATO, G. et al. Rearing veal calves with respect to animal welfare: effects of group housing and solid feed supplementation on growth performance and meat quality. Livestock Production Science, Amsterdam, v.75, n.3, p.269-280, 2002.

ZDANOWICZ, M. et al. Bacterial populations on teat ends of dairy cows housed in free-stalls and bedded with either sand or sawdust. Journal of Dairy Science, Champaign, v.87, p.16941701, 2004 